\title{
PROKLA-Redaktion
}

\section{Editorial: Konflikte um den Wohlfahrtsstaat}

Nicht erst jetzt ist der Wohlfahrtsstaat ins Gerede gekommen: Die Rede von den »leeren Kassen«, den »überzogenen Ansprüchen《 oder der »Überforderung des Sozialstaats« gehen ins dritte Jahrzehnt, an den Stammtischen ebenso wie in der wissenschaftlichen und politischen Öffentlichkeit. Genauso lange sind auch bereits kritische Stimmen gegen die »Rotstiftpolitik «, den »sozialen Kahlschlag《 oder die »Demontage « des bis dahin so erfolgreichen bundesrepublikanischen Konsensmodells zu vernehmen. Dabei mußte oftmals der Eindruck entstehen, die oppositionelle Rhetorik sei inzwischen heiser geworden. Wenn Jahre über Jahre mit pathetischer Geste eine stete Wiederholung von »Kahlschlag《 und »Demontage beschworen wurde, schien der Wohlfahrtsstaat mehr als einmal an sein Ende gekommen zu sein und so etwas wie eine mehrfach bestattete Leiche darzustellen. Anders als in zahlreichen apokalyptischen Visionen vorhergesagt, erfolgte der Abbau der wohlfahrtsstaatlichen Strukturen allerdings nicht in rasender Talfahrt, sondern in einem eher gemäßigten Tempo und in widersprüchlichen Formen. Dennoch hat er inzwischen im Großen und Ganzen zweifellos stattgefunden, und die früher nicht immer fundierte Aufgeregtheit der Kritiker dieser Entwicklungen ist sehr viel differenzierteren Analysen gewichen.

Linke Diagnosen und Prognosen schossen zwar mitunter übers Ziel hinaus, erkannten aber durchaus zutreffend die Grundtendenz einer politisch gewollten und durchgesetzten Verschärfung der sozialen Polarisierung. Demgegenüber halten zahlreiche Postulate des konservativen und des liberalen Diskurses einer Überprüfung vor dem Hintergrund realer Entwicklungen nicht stand, was ihre Verankerung im öffentlichen Bewußtsein jedoch in keiner Weise verhindert hat. Zwar wissen mittlerweile auch Rentner, Disco-Besucher oder andere Menschen, die nicht regelmäßig die Publikationen von Wirtschaftsforschungsinstituten verfolgen, daß große Konzerne ihre Entlastung bei den Unternehmenssteuern $\mathrm{zu}$ allen möglichen Zwecken genutzt haben, aber sicherlich nicht dazu, neue Arbeitsplätze zu schaffen. Dennoch gilt die massive staatliche Umverteilung von unten nach oben mehrheitlich keineswegs als skandalös, sondern immer noch durch wirtschaftliche Rationalität gerechtfertigt. Der Bundesregierung, den Arbeitgeberverbänden 
und den ihnen nahestenden »Experten« gelang es bisher stets, alle empirischen Befunde, die dem Zielkatalog der Deregulierung entgegenstanden, souverän zu ignorieren - ob es um die angeblich horrend gestiegene Sozialleistungsquote, die »Kostenexplosion" im Gesundheitswesen, die allgemein $» z \mathrm{zu}$ hohen« Löhne und Lohnnebenkosten oder die vermeintlich allzu bequemen und arbeitsunwilligen Arbeitslosen ging. Argumente, die oftmals auf tönernen FüBen standen, wurden als nüchterne und realitätsgerechte Interpretationen der Krise der öffentlichen Finanzen und des Arbeitsmarktes etabliert.

So läuft vor allem die neuere Diskussion um den Sozialstaat, wie Heiner Ganßmann zeigt, mit einer ausgiebigen Verwendung von falschen Etiketten ab. Während der Bundeskanzler sich in der Nachfolge Bismarcks als historische Lichtgestalt feiern läßt, der die Vollendung der deutschen Einheit zu verdanken sei, werden die Kosten des ökonomisch fehlgesteuerten Vereinigungsprozesses auf dem falschen Konto verbucht: der Sozialstaat sei unerträglich teuer und verhindere die Konkurrenzfähigkeit der Bundesrepublik auf dem Weltmarkt. Dabei folgt aber bei den so begründeten Einsparungen bei den sozialen Leistungen in der gegenwärtigen wirtschaftlichen Situation nur eine Abwärtsspirale, in der sinkende Steuereinnahmen zu mehr Staatsverschuldung und zu neuen Sparzwängen führen.

Aber nicht die gescheiterte $\gg$ Angebotspolitik«, sondern der Sozialstaat soll für Massenarbeitslosigkeit und Staatsverschuldung verantwortlich sein. Daher wird auch munter an einer bisher zentralen Institution des Sozialstaats reformiert, der Gesetzlichen Krankenversicherung. Tomas Steffens macht in seinem Beitrag deutlich, daß das, was hier als überfällige Reform zur Kostensenkung verkauft wird, keineswegs auf eine rationellere gesellschaftliche Ressourcenverwendung hinausläuft, sondern auf eine weitgehende Privatisierung des Krankheitsrisikos. Das »Gut« Gesundheit können sich ärmere Schichten dann eben nur in einem geringeren Maße leisten als diejenigen, die auch schon mit den übrigen Gütern reich gesegnet sind.

$\mathrm{Daß}$ Armut in den letzten zehn, fünfzehn Jahren in der Bundesrepublik stark zugenommen hat und noch weiter zunimmt, läßt sich inzwischen auch von konservativer Seite nicht mehr bestreiten. In Frage steht allerdings, um welche Form von Armut es sich dabei vorwiegend handelt. So kam die »dynamische Armutsforschung « zu dem Ergebnis, daß Armut in den letzten Jahrzehnten zu einem lebensgeschichtlichen Übergangsphänomen geworden sei: sie träfe zwar mehr Menschen als früher, aber kaum noch jemanden dauerhaft. Die Armut als "schicksal« in einer Klassengesellschaft habe somit ihren Schrecken verloren. Diese von der Bundesregierung mit Genugtuung aufgenommene Diagnose der $»$ Entwarnung « steht nicht nur im Widerspruch zur Alltagserfahrung jedes U-Bahn-Benutzers in einer Stadt wie Berlin, sondern auch zu einer ganzen Reihe von empirischen Studien. Peter Bremer und Norbert Gestring diskutieren daher in 
ihrem Beitrag, ob sich das in den USA entwickelte Konzept der verfestigten Armut einer »Underclass « auch auf die Bundesrepublik übertragen läßt. Zumindest Teile der ausländischen Wohnbevölkerung in deutschen Städten kommen in Zukunft als Kandidaten für eine solche »Underclass « in Frage, da sie von mehrfachen Diskriminierungen (auf dem Arbeitsmarkt, dem Wohnungsmarkt sowie durch eine Reihe rechtlicher Regelungen) betroffen sind.

Angesichts immer neuer wohlfahrtsstaatlicher Kürzungsrunden sowie einer ganz unverblümten Regierungpolitik zugunsten der Arbeitgeberseite, stellt sich die Frage nach den Möglichkeiten gewerkschaftlicher Gegenwehr. Daß sich diese zwangsläufig in einer »Globalisierungsfalle« befindet, wird von Jürgen Hoffmann vehement bestritten: nicht nur sei die $»$ Globalisierung « differenzierter zu betrachten, auch müßten die Gewerkschaften neue Formen branchenübergreifender Politik entwickeln und sich auf nationaler wie auf internationaler Ebene für eine sozial und ökologisch orientierte $\gg$ Re-Regulierung« engagieren.

$\mathrm{Zu}$ pessimistischeren Prognosen kommt Michael Wendl. Sowohl dem »Bündnis für Arbeit« als auch der Diskussion um die Öffnung der Flächentarifverträge liege eine »angebotspolitische« Wende gewerkschaftlicher Politik zugrunde, die von der fragwürdigen Hoffnung geleitet sei, daß Lohnverzicht neue Arbeitsplätze schaffen würde. Auch durch verteilungspolitische Niederlagen würden die Gewerkschaften kaum von ihrer vor allem auf Konsens mit den Arbeitgebern orientierten Politik abrücken.

Während neoklassische Scheinplausibilitäten bis in die Formulierung gewerkschaftlicher Politik vorgedrungen sind, scheint der von Regierungen und Arbeitgebern betriebene Abbau solidarischer und sichernder Regelungen in letzter Zeit allerdings auf einen stärkeren Widerstand der Betroffenen zu stoBen: Auf sehr verschiedene Art haben Arbeiter und Arbeiterinnen in Frankreich, Deutschland und Korea gleichlautende Signale dafür gesetzt, daß sie den neoklassischen Alltagsweisheiten möglicherweise nicht in alle Ewigkeit trauen werden.

$* * *$

Außerhalb des Schwerpunkts, aber nicht ohne Bezug zu ihm, erscheint in diesem Heft ein Beitrag von Michael Mann, der sich kritisch mit der These von der abnehmenden Bedeutung der Nationalstaaten im Zeitalter der Globalisierung auseinandersetzt. Der Aufsatz von Uli Jähner zeigt die nicht wahrgenommene Aktualität eines Klassikers der politischen Theorie, Kants Friedensschrift. Indem es hier um das Verhältnis von Bürgern zu Staaten und von Staaten untereinander geht, schließt dieser Beitrag an das Thema des letzten Heftes an - »Fragmentierte Staatsbürgerschaft $\ll$. 